\title{
Support and stay: an innovative community service for the elderly confused
}

\author{
Paul Whitby, Senior Clinical Psychologist; Joan Rule, Consultant Psychogeriatrician; \\ and JiLL JoomRatTY, Senior Nurse, Tonna Hospital, near Neath, \\ West Glamorgan SA11 3LX
}

\section{Background}

In the psycho-geriatric field there is great concern that as the number of elderly people in the population rises so the number of those suffering from dementia will increase. Services are already stretched and innovatory changes are needed to ensure that this large group of people receive adequate care.

The task before us is to ensure that people who wish to remain at home receive enough support, either directly, or indirectly through their relatives, to make this option safe, comfortable and dignified. There is also persistent difficulty in recruiting and retaining qualified nurses in this field, which is expected to get worse. In our part of West Glamorgan we have employed nursing assistants (NAs) to provide basic nursing care in the community, thus freeing the trained community psychiatric nurses (CPNs) for other duties. The scheme is titled 'Support and Stay'.

\section{Present state}

Cover is provided between $7 \mathrm{pm}$ and 9 am for seven days a week (including Bank Holidays and Christmas Day). The CPNs work 9 am-5 pm weekdays. At other times cover is provided by the senior nurse on the in-patient ward and an on-call psychiatric registrar. The consultant and senior nurse are available out of working hours for advice.

Between two and four NAs are on duty each morning shift and two each evening shift. The busiest times of the day are early morning and the later evening when the NAs are getting patients out of bed, washed and dressed in the one case and undressed and put to bed in the other. At other times the NAs perform a variety of tasks. They may assist with or solely provide any necessary care; for example, they may sit with a patient while a carer goes out, or they may take the patient shopping while the carer has a break. They may check that a patient has taken his or her prescribed medication, or they may bring that patient up to hospital for an out-patient appointment.

\section{Organisation}

\section{Referral and discharge}

All patients are referred to the CPNs for the Support and Stay (SaS) service by the consultant. Discharge from the SaS scheme is by transfer to another part of the psychogeriatric service, to residential care, or by death.

\section{Responsibility for care and supervision of unqualified stafi}

Each CPN holds nursing responsibility for a case load of patients. Medical responsibility is held by the consultant and supervision is provided in regular review meetings. The consultant indicates the amount of care needed and problem areas particularly pressing. The CPN then draws up a Nursing Care Plan upon which the intensity and duration of visits by the NAs and the nature of the tasks they will perform is based. Regular team meetings are held in which these Care Plans are reviewed. As a further method of supervision, patients who are being visited by the NAs are also visited by the CPN regularly and will also see a member of medical staff at intervals.

\section{Activity}

In November 1989 there were eight nursing assistants who visited 92 patients; 984 separate calls were made. Calls per patient ranged from 1 to 59 in the month. The average number was just over 10 . A more informative statistic is that the most dependent 14 patients received 501 visits during the month (i.e. $15 \%$ of patients received $51 \%$ of visits).

\section{Patient characteristics}

Thirty-five patients were assessed using the Behaviour Rating Scale from the Clifton Assessment Procedures for the Elderly (Pattie \& Gilleard, 1979). The sample included all patients on the case-load of one CPN. The CAPE-BRS was completed by the NAs. 
TABLE I

Levels of dependency of patients

\begin{tabular}{llr}
\hline & CAPE calegory & $n$ \\
\hline A & Independent elderly & 2 \\
B & Low dependency & 4 \\
C & Medium dependency & 10 \\
E & High dependency & 12 \\
Maximum dependency & 7 \\
\hline & Total & 35 \\
\hline
\end{tabular}

The categories of dependency to which these patients were assigned are shown in Table I.

This shows the $\mathrm{SaS}$ is reaching patients with a wide range of dependency needs. Many of these people are typical of those found in Social Services residential and nursing homes and in hospital psychogeriatric wards.

\section{Carer survey}

We asked carers if they thought it would be possible for them to continue to care for their relative at home without the SaS. The results are shown in Table II.

Carers were also asked to comment on the scheme. Most were very favourable yet two negative themes recurred. Firstly, several carers said they would prefer to receive visits at regular times each day. The present system whereby busy NAs fit in their visits whenever possible is less than perfect. Secondly, evening calls to prepare a patient for bed were being made too early. Both these criticisms reflect how busy the NAs are in early mornings and evenings.

\section{Comment}

\section{Is the scheme meeting its aims?}

In terms of numbers the Support and Stay scheme appears successful; 92 patients were visited by the NAs in November 1989. This is more than would have been possible than when the CPNs worked unassisted. It is difficult to estimate how many would otherwise have required residential care had the SaS not been available. However, $55 \%$ of those carers who responded to our survey said they could not continue without this help and a further $28 \%$ said they could continue only with great difficulty. Of the 35 patients, 19 assessed by the CAPE-BRS (54\%) belonged to the High or Maximum Dependency categories and as such are typical of the elderly seen in nursing homes and psychogeriatric wards. A further ten were Medium Dependency. These are
TABLE II

Carers' perception of their need for the service

Question 9: Without the support and stay service would you be able to keep your relative at home?

$\begin{array}{llr}\text { 1. } & \text { Yes, definitely } & 2 \\ \text { 2. } & \text { Yes, probably } & 3 \\ \text { 3. } & \text { Yes, just about } & 1 \\ \text { 4. } & \text { Yes, with great difficulty } & 11 \\ \text { 5. } & \text { No, probably not } & 8 \\ \text { 6. } & \text { No, definitely not } & 14\end{array}$

good indicators that $\mathrm{SaS}$ is reaching the appropriate people.

The flexibility of the scheme is one of its strengths. A few patients receive intense care from the NAs and the majority receive rather less. As a patient's condition deteriorates the level of care is increased. This flexibility also extends to sudden crises when staff may increase support at short notice.

\section{What is the quality of care being provided?}

Day to day quality control rests with the CPNs. The NAs are supervised by the CPNs who visit patients and carers regularly, and by team meetings held to discuss patients. The CPNs also generally make themselves readily available to the NAs and encourage requests for advice, assistance or opinion.

One other guarantee of quality is that the service is provided in peoples' own homes. It is commonly remarked that carers do not hesitate to make their opinions known!

How does the Support and Stay scheme fit in with the rest of the psychogeriatric service?

The SaS has provided the psychogeriatric team with a flexible and far-reaching facility. When aiming for a service which is responsive to patients needs, it is essential to provide a variety of options of care from which the team, the carers, and often the patient, can select the most appropriate.

Other features of our service are: in-patient beds (long-stay, assessment, respite and relief), a day hospital, eight day-centres, out-patient clinics and a community nursing service over and above the Support and Stay scheme. Communications between the parts of the service are efficient and it has proved easy to integrate the SaS into the existing service. People discharged from the in-patient ward can be offered assistance at home with little delay. People seen in out-patients can be seen swiftly by a CPN who can arrange visits by the NAs. In practice it is easy for patients to use the various aspects of the service as and when necessary. 


\section{Conclusion}

Eighteen months experience with unqualified NAs in community work with the confused elderly has been a great success. We were able to recruit high calibre staff and in future it may be that more training and supervision will be called for. Training and supervision are the keys to successful utilisation of unqualified staff. This has required a change in working practice of our CPNs who traditionally are free of these duties and are more used to working independently.

Two aspects of our NAs' duties should be stressed. Firstly, they are part of a psychiatric team and are concerned not simply with physical tasks. They are expected to provide emotional and psychological support of a simple kind. Secondly, they belong to a multidisciplinary team and have ready access to a wide range of expertise. These two aspects of the NAs' role mark them out as quite different both from other non-psychiatric NHS colleagues and from local authority home care workers.

Our conclusion is that given the right kind of support and guidance unqualified staff can perform an essential role in the care of the elderly mentally ill. Given the growing shortage of trained nurses it would seem likely that this model of working will become more common in the future.

\section{Reference}

Pattie, A. H. \& Gilleard, C. J. (1979) Manual of the Clifton Assessment Procedures for the Elderly (CAPE). Sevenoaks: Hodder \& Stoughton.

\title{
The development of a community support service for the chronically mentally ill in an inner city health district
}

\author{
StePhen Colgan, Senior Registrar in Psychiatry; and Keith Bridges, Consultant \\ Psychiatrist, Department of Psychiatry, Rawnsley Building, Manchester Royal \\ Infirmary, Oxford Road, Manchester M13 9WL
}

Community support teams are an important component of services for the chronically mentally ill who do not need to remain in hospital. In the autumn of 1989 the Department of Psychiatry in Central Manchester established a small team with a remit to develop such a service. The team is made up of four nursing staff (a charge nurse, staff nurse, SEN and a nursing auxiliary) with additional support provided by two occupational therapists, a clinical psychologist, consultant psychiatrist and senior registrar in rehabilitation psychiatry. This paper will briefly describe some of the experiences and problems that the service has encountered so far and some of the solutions that have been explored.
The philosophy of the service is to help clients live in the community as independently as possible with a reasonable quality of life. Patients seen by the team are referred to as 'clients' as part of a process to demedicalise their lives. Each one is allocated a 'keyworker' who carries out an initial assessment of social needs and identifies the type of help and support that is required. If these can be provided by the service, goals are formulated in consultation with the client and targets agreed. This management plan is then reviewed on a regular basis at team meetings to which the client is invited to attend. Following the initial assessment, the key worker has responsibility for co-ordinating 